\title{
G-CSF receptor truncations found in SCN/AML relieve SOCS3-controlled inhibition of STAT5 but leave suppression of STAT3 intact
}

\author{
Gert-Jan M. van de Geijn, Judith Gits, Lambertus H. J. Aarts, Claudia Heijmans-Antonissen, and Ivo P. Touw
}

Truncated granulocyte colony-stimulating factor receptors (G-CSF-Rs) are implicated in severe congenital neutropenia (SCN) and the consecutive development of acute myeloid leukemia (AML). Mice expressing G-CSF-R truncation mutants (gcsfr-d715) show defective receptor internalization, an increased signal transducer and activator of transcription 5 (STAT5)/STAT3 activation ratio, and hyperproliferative responses to G-CSF treatment. We determined whether a lack of negative feedback by suppressor of cytokine signaling (SOCS) proteins contributes to the signaling abnormalities of
G-CSF-R-d715. Expression of SOCS3 transcripts in bone marrow cells from G-CSF-treated gcsfr-d715 mice was approximately $60 \%$ lower than in wild-type (WT) littermates. SOCS3 efficiently suppressed STAT3 and STAT5 activation by WT G-CSF-R in luciferase reporter assays. In contrast, while SOCS3 still inhibited STAT3 activation by G-CSF-R-d715, STAT5 activation was no longer affected. This was due mainly to loss of the SOCS3 recruitment site Tyr729, with an additional contribution of the internalization defects of G-CSF-R-d715. Because Tyr729 is also a docking site for the Src homol- ogy 2-containing protein tyrosine phosphatase-2 (SHP-2), which binds to and inactivates STAT5, we suggest a model in which reduced SOCS3 expression, combined with the loss of recruitment of both SOCS3 and SHP-2 to the activated receptor complex, determine the increased STAT5/STAT3 activation ratio and the resulting signaling abnormalities projected by truncated G-CSF-R mutants. (Blood. 2004;104:667-674)

(c) 2004 by The American Society of Hematology

\section{Introduction}

Granulocyte colony-stimulating factor (G-CSF) receptor is the major regulator of neutrophil production, both under steady-state conditions and during stages of bacterial infections. ${ }^{1-3}$ G-CSF exerts its activity via a receptor (G-CSF-R) of the hematopoietin receptor superfamily. ${ }^{4,5}$ Typical of this class of receptors, G-CSF-R has no intrinsic kinase activity but recruits cytoplasmic tyrosine kinases of both the Janus kinase (Jak) and Src kinase families and activates signal transducer and activator of transcription (STAT) proteins. ${ }^{6-11}$ G-CSF activates STATs 1,3 , and $5 .{ }^{12-14}$ Whereas the contribution of STAT1 to G-CSF responses remains unclear, STAT3 has been implicated in G-CSF-mediated growth arrest preceding differentiation, while activation of STAT5 has been linked to proliferation and survival signaling. ${ }^{15-17}$ Four tyrosine residues (Tyr704, Tyr729, Tyr744, and Tyr764) in the G-CSF-R carboxy-terminus are involved in the recruitment of signaling molecules, such as the adapter molecules growth factor receptorbound protein 2 (Grb2) and Src homology and collagen protein (Shc) of the p21Ras-mitogen-activated protein (MAP) kinase pathway, and the Src homology 2-containing protein tyrosine phosphatase-2 (SHP-2). ${ }^{18-20}$ In addition, activation of STAT3 depends on its recruitment to the G-CSF-R via tyrosines 704 or 744. ${ }^{20-22}$ At higher G-CSF concentrations, STAT3 can also be activated in a tyrosine-independent way via the G-CSF-R Cterminus. ${ }^{22,23}$ In contrast, activation of STAT1 and STAT5 is achieved via the membrane-proximal region of G-CSF-R and does not require receptor tyrosine residues. ${ }^{13,17}$
In approximately $20 \%$ of patients suffering from severe congenital neutropenia (SCN), G-CSF-R mutations are found that result in the expression of a G-CSF-R with a truncated C-terminus. ${ }^{24,25}$ These patients have an increased risk of developing acute myeloid leukemia (AML). ${ }^{25,26}$ Activation of a G-CSF-R mutant truncated at amino acid 715 (G-CSF-R-d715) causes a hyperproliferative response in $32 \mathrm{D}$ cells, without induction of neutrophilic differentiation. ${ }^{27}$ Mice with a targeted G-CSF-R-d715 mutation show various degrees of neutropenia, and their myeloid precursors react to G-CSF administration with hyperproliferation, resulting in a sustained neutrophilia. ${ }^{28,29}$ Interestingly, transgenic (Tg) mice overexpressing G-CSF-R mutants truncated at amino acids (aa's) 718 and 731 demonstrated increased susceptibility to infection with Staphylococcus aureus, suggesting that production of functional neutrophils is compromised in these animals. ${ }^{30}$ Indeed, these Tg mice had only one third of the peripheral neutrophil levels of wild-type (WT) controls, and their bone marrow showed increased percentages of immature myeloid cells.

Functional analysis of truncated receptors revealed that a number of properties are altered compared with WT G-CSF-R. Ligand-induced internalization of G-CSF-R-d715 is severely affected owing to the loss of 2 distinct motifs in the receptor C-terminus that are important for internalization. ${ }^{27,31,32}$ G-CSF-Rd715 also has a somewhat reduced ability to activate STAT3, possibly owing to the loss of the STAT3 recruitment site Tyr744 and the receptor C-terminus. ${ }^{22,31}$ In contrast, activation of STAT5 is
From the Institute of Hematology, Erasmus Medical Center, Rotterdam, The Netherlands.

Submitted August 22, 2003; accepted March 22, 2004. Prepublished online as Blood First Edition Paper, April 6, 2004; DOI 10.1182/blood-2003-08-2913.

Supported by the Dutch Cancer Society "Koningin Wilhelmina Fonds."

Reprints: Ivo P. Touw, Erasmus MC, Institute of Hematology, Rm Ee 1330c,
PO Box 1738, 3000 DR Rotterdam, The Netherlands; e-mail: i.touw@ erasmusmc.nl.

The publication costs of this article were defrayed in part by page charge payment. Therefore, and solely to indicate this fact, this article is hereby marked "advertisement" in accordance with 18 U.S.C. section 1734.

(C) 2004 by The American Society of Hematology 
strongly increased and is sustained after removal of G-CSF, suggesting a prominent role for the $\mathrm{C}$-terminus in mediating negative feedback on STAT5 activation. ${ }^{17,27,31}$ Although the defective internalization properties of truncated G-CSF-R forms contributed significantly to their sustained signaling function, it was also clear that this did not fully explain these findings. ${ }^{27,32}$ In particular, the differential effects of receptor truncations on the kinetics of STAT3 versus STAT5 activation remained unclear.

Suppressor of cytokine signaling (SOCS) proteins are involved in the down-regulation of signaling from a number of hematopoietic growth factor receptors, including G-CSF-R. ${ }^{33-36}$ A conserved $\mathrm{SH} 2$ domain and a C-terminal SOCS box are characteristic for the SOCS family (reviewed in Krebs and Hilton, ${ }^{37}$ Kile and Alexander, ${ }^{38}$ and Alexander ${ }^{39}$ ). The expression of most SOCS genes is controlled by STAT transcription factors. ${ }^{40-45}$ SOCS proteins therefore act in a classical negative feedback loop to suppress cytokine signaling. Three distinct inhibitory mechanisms have been linked to SOCS proteins. Cytokine-inducible $\mathrm{SH} 2$-containing protein (CIS), founding member of the family, inhibits activation of STAT5 by competing for STAT5 recruitment to phosphotyrosine motifs in, for example, the growth hormone receptor (GHR) and the erythropoietin receptor (EpoR). ${ }^{46,47}$ SOCS1 and SOCS3, on the other hand, directly suppress Jak kinase activity by means of a kinase inhibitory region (KIR). ${ }^{48-50}$ Upon recruitment to the signaling complex via the SH2 domain of SOCS, the KIR mediates inhibition by blocking access of both adenosine triphosphate (ATP) and substrate to their binding sites in the catalytic groove of Jak2. ${ }^{49-53}$ Finally, SOCS proteins are also thought to down-regulate signaling via SOCS box-mediated targeting of signaling proteins for proteasomal degradation. ${ }^{54,55}$ An important difference between SOCS1 and SOCS3 relates to how they are recruited into activated receptor complexes. Whereas the SH2 domain of SOCS1 has a high affinity for, for example, phosphorylated Tyr1007 in the Jak homology 1 (JH1) domain of Jak2, the affinity of the SH2 domain of SOCS3 for this residue is much lower. ${ }^{49,52,56}$ Instead, SOCS3 is recruited with high affinity to phosphotyrosine-based motifs in certain receptors and then subsequently inhibits Jak activity via its KIR. ${ }^{34,35,48,57-59}$ Importantly, for a number of cytokine receptors, it has been established that the protein tyrosine phosphatase SHP-2 and SOCS3 dock to identical tyrosine-based motifs with comparable affinities. ${ }^{57-60}$

Studies in SOCS-deficient mice have demonstrated major physiologic roles for SOCS proteins in controlling the levels of cytokine signaling in both nonhematopoietic and hematopoietic cells. ${ }^{61-72}$ G-CSF induces the expression of SOCS1, SOCS2, SOCS3, and CIS in hematopoietic cells, ${ }^{35,73}$ but only SOCS1 and SOCS3 appeared to inhibit G-CSF-induced STAT activation. ${ }^{74}$ In 2 independent studies, Tyr729 of G-CSF-R was identified as the major recruitment site for SOCS3. ${ }^{35,36}$ Recently, it was reported that SOCS3 is a key negative regulator of G-CSF-induced neutrophil production in vivo. ${ }^{75,76}$

In the present study, we investigated to what extent and by which mechanism(s) truncation of the G-CSF-R C-terminus, as found in SCN, affects the negative feedback regulation of G-CSF signaling by SOCS3. We show that truncation of the G-CSF-R completely relieved the inhibitory effects of SOCS3 on activation of STAT5. In striking contrast, the suppressive effects of SOCS3 on G-CSF-R-d715-induced activation of STAT3 were hardly affected by the truncation. These findings provide a new mechanistic explanation for the increased ratio of STAT5/STAT3 activation in gcsfr-d715 mice, which has previously been linked to the shift in the proliferation/differentiation balance in the myeloid progenitor cell compartment found in these animals. ${ }^{31}$

\section{Materials and methods}

\section{Expression constructs}

The constructs of human G-CSF-R WT, d715, Tyr729Phe, d735, d749-769, d715-735, and the green fluorescent protein (GFP)-G-CSF-R fusions in the pBabe vector ${ }^{77}$ have been described before ${ }^{18,20,32,36}$ (Figure 1A). The d735Phe and d749-769Phe mutants were generated from mutant Tyr729Phe by, respectively, introduction of a stop codon or deletion of aa's 749-769 with a site-directed mutagenesis kit according to the manufacturer's instructions (Stratagene, La Jolla, CA). Expression constructs of myctagged SOCS1 and SOCS3 in pcDNA3 were a gift from A. Yoshimura. ${ }^{56}$ For expression of SHP-2, hemagglutinin (HA)-tagged human SHP-2 was cloned into the EcoRI-XhoI sites of expression vector pSG5. ${ }^{78}$ For expression of STAT5, pME18S-STAT5B was used. ${ }^{79}$

\section{Bone marrow cells and isolation of RNA}

WT and $g c s f r-d 715$ mice ${ }^{28}$ were stimulated daily for 4 days with G-CSF or received solvent only. Each experimental group contains 2 mice of each genotype. Bone marrow cells were isolated, resuspended in TRIzol RNA extraction reagent (Invitrogen, Breda, The Netherlands), snap frozen, and stored at $-80^{\circ} \mathrm{C} .{ }^{28}$ For the in vitro stimulation, bone marrow cells were harvested from 2 WT and 2 gcsfr-d715 mice that had not been treated with G-CSF. Cells were cultured for 1 hour in Hanks balanced salt solution (HBSS; Invitrogen) with 5\% fetal calf serum (FCS); nonadherent cells were taken and starved for 4 hours in RPMI (Invitrogen) plus $0.5 \%$ bovine serum albumin (BSA). Cells were stimulated for the indicated periods with G-CSF $(100 \mathrm{ng} / \mathrm{mL})$ and resuspended in Trizol. RNA was isolated according to the manufacturer's instructions and subsequently treated with DNAse to remove genomic DNA. DNAse treatment of $5 \mu \mathrm{g}$ RNA was performed in DNAse buffer (40 mM Tris-HCl [tris(hydroxymethyl)aminomethane-HCl],
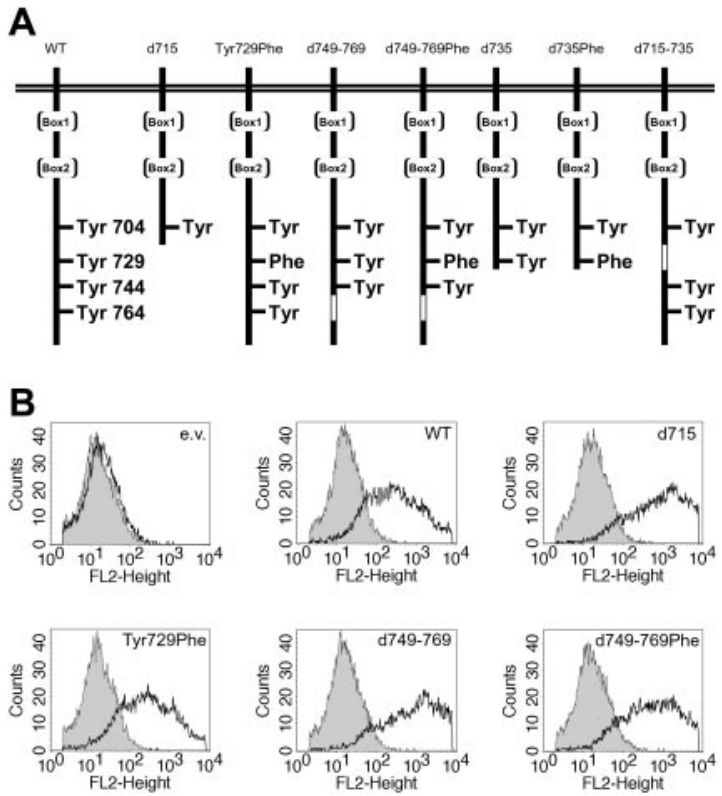

Figure 1. Expression of WT and mutant G-CSF receptors. (A) Schematic representation of the intracellular domain of the G-CSF receptor and mutants. Boxes 1 and 2 represent subdomains conserved in the hematopoietin receptor superfamily. The open box indicates the deleted region in G-CSF-R-d749-769 and G-CSF-R-d715735. (B) Flow cytometric analysis of the expression levels of the different G-CSF-R forms used in luciferase reporter assays. Open histograms indicate cells stained with biotinylated G-CSF-R antibodies and phycoerythrin-conjugated streptavidin (SA-PE); shaded histograms, cells stained with SA-PE only; and e.v., cells transfected with empty vector. 
$\mathrm{pH} 7.5 ; 6 \mathrm{mM} \mathrm{MgCl} 2 ; 2 \mathrm{mM} \mathrm{CaCl} 2)$ with $10 \mathrm{U}$ DNAse I (Stratagene) for 1 hour at $37^{\circ} \mathrm{C}$.

\section{Quantitative reverse-transcriptase PCR (RT-PCR)}

To generate cDNA, $1 \mu \mathrm{g}$ RNA was denatured at $65^{\circ} \mathrm{C}$ for 5 minutes followed by 10 minutes on ice. After addition of first-strand buffer $(250 \mathrm{mM}$ Tris- $\mathrm{HCl}, \mathrm{pH} 8.3 ; 375 \mathrm{mM} \mathrm{KCl} ; 15 \mathrm{mM} \mathrm{MgCl} 2$ ) with deoxynucleoside triphosphates (dNTPs; $1 \mathrm{mM}$ final concentration), dithiothreitol (DTT; 1 $\mathrm{mM}$ final concentration), $4 \mu \mathrm{g}$ random hexamers (Amersham Pharmacia, Uppsala, Sweden), 40 U RNasin, and 200 U Superscript II reverse transcriptase (Invitrogen), the reaction was incubated at $42^{\circ} \mathrm{C}$ for 2 hours. The cDNA was diluted 1:10, 1:30, and 1:60 for SOCS1, SOCS3, and ribonuclease inhibitor, respectively, before polymerase chain reaction (PCR) amplification. Primers used for amplification for SOCS1 were as follows: FTMSOCS1, 5'-tggtagcacgcaaccaggtg; and RTMSOCS1, 5' tggcgaggacgaagacgag. Primers used for SOCS3 were FTMSOCS3, 5'tcaagaccttcagctccaa; and RTMSOCS3, 5'-tcttgacgctcaacgtgaag. Primers for murine ribonuclease inhibitor were forward 5 'tccagtgtgagcagctgag, and reverse 5 '-tgcaggcactgaagcacca. For the quantitative real-time PCR, Taqman technology was used (Model 7900 sequence detector; PE Applied Biosystems, Foster City, CA). The reactions were performed in a $25 \mu \mathrm{L}$ vol of a mixture containing $2 \mu \mathrm{L}$ of the respective cDNA dilution, primers at 5 $\mu \mathrm{M}$, and $12.5 \mu \mathrm{L}$ of $2 \times$ SYBR green PCR Master mix (PE Applied Biosystems) containing Amplitaq Gold DNA polymerase, reaction buffer, dNTP mix with uridine $5^{\prime}$-triphosphate (UTP), and the double-stranded DNA-specific fluorescence dye SYBR green I. The PCR program used was 1 cycle of 2 minutes at $50^{\circ} \mathrm{C}, 1$ cycle of 10 minutes at $95^{\circ} \mathrm{C}, 45$ cycles of denaturation for 15 seconds at $95^{\circ} \mathrm{C}$, annealing for 30 seconds at $62^{\circ} \mathrm{C}$, and extension for 30 seconds at $62^{\circ} \mathrm{C}$. To determine the expression levels, samples were tested in duplicate, and the average values of the threshold cycle $(\mathrm{Ct})$ were used for quantification. To quantify the relative expression of SOCS1 and SOCS3, the Ct values were normalized for endogenous reference $\left(\Delta \mathrm{Ct}=\mathrm{Ct}_{\text {SOCS }}-\mathrm{Ct}_{\text {ribonuclease inhibitor }}\right)$ and compared with a calibrator, by means of the $\Delta \Delta \mathrm{Ct}$ method $\left(\mathrm{Ct}=\mathrm{Ct}_{\text {sample }}-\mathrm{Ct}_{\text {Calibrator }}\right)$. As calibrator for G-CSF stimulation in vivo, we used the expression in WT bone marrow of unstimulated mice. As calibrator for the in vitro stimulation, we used expression after 4 hours of starvation.

\section{Luciferase assays}

Luciferase assays were performed as described previously. ${ }^{36}$ In short, HEK293 cells, grown in 24-well plates, were transfected by the calcium phosphate precipitation method with a mixture of the following plasmids. For STAT5 luciferase experiments, we used expression vector pME18SSTAT5, a $\beta$-casein-derived STAT5 luciferase reporter plasmid, a $\beta$-galactosidase expression plasmid pRSVLacZ, a pBabe construct with WT or mutant G-CSF-R (Figure 1A), and different amounts of pCDNA3 with myc-tagged SOCS or empty pcDNA3 (Invitrogen). For the STAT3 luciferase experiments pME18S STAT5 was replaced by pcDNA3 vector, and an m67-derived STAT3 luciferase reporter was added instead of the STAT5 reporter. A volume of $100 \mu \mathrm{L}$ calcium phosphate precipitate in a total of 2 $\mu \mathrm{g}$ DNA was added to each well. With the exception of SOCS3, $400 \mathrm{ng}$ DNA for each construct was added per well. Different amounts of pcDNA3-SOCS3 were added, supplemented with empty pcDNA3 vector up to $400 \mathrm{ng}$. After 24 hours, the cells were starved overnight in Dulbecco minimum essential medium (DMEM) plus penicillin/streptomycin (pen/ strep) plus $0.1 \%$ BSA. The next day, the cells were stimulated with 250 $\mathrm{ng} / \mathrm{mL}$ G-CSF for 6 hours, lysed, and assayed for luciferase activity with the use of Steady-Glo reagents (Promega, Madison, WI). In parallel, the transfection efficiency was determined by means of lacZ staining. Luciferase activity levels were corrected for transfection efficiency with the use of $\beta$-galactosidase expression levels. All experiments were performed in triplicate. Fold induction by G-CSF was calculated and set at $100 \%$ in the absence of SOCS. For inhibitor studies, the Src inhibitor PP-2, the Jak inhibitor WHI-P154 (Calbiochem, San Diego, CA), or dimethyl sulfoxide (DMSO) as a solvent control was added to the cells 1 hour prior to G-CSF stimulation. Unless stated otherwise, data were analyzed by means of analysis of variance (ANOVA). To compare G-CSF-R expression levels, transfected cells were stained with biotinylated mouse antihuman G-CSF-R antibody (Pharmingen, San Diego, CA) followed by SA-PE (Caltag Laboratories, Burlingame, CA), and analyzed by flow cytometry (FACSCalibur; Becton Dickinson, Sunnyvale, CA). As shown in Figure 1B, G-CSF-R expression is detectable with increased (less than $1 \log$ unit) expression of the internalization-defective mutants (G-CSF-R-d715, d749-769, and d749$769 \mathrm{Phe})$. As previously reported, this difference is due mainly to decreased spontaneous internalization of these mutants in the absence of G-CSF in nonmyeloid cells. ${ }^{32}$

\section{Immunoprecipitations and Western blotting}

Phoenix E cells (a gift from G. Nolan, Stanford, CA) were transfected with G-CSF-R, SHP-2, and, in the case of the SHP-2-STAT5 coimmunoprecipitations, with the STAT5 expression construct as well. After 24 hours, the medium was replaced by DMEM plus $0.1 \%$ BSA. The next day, cells were stimulated for 10 minutes with G-CSF, washed twice with cold phosphatebuffered saline (PBS), and lysed in lysis buffer containing $20 \mathrm{mM}$ Tris- $\mathrm{HCl}$, $\mathrm{pH} 8.0 ; 137 \mathrm{mM} \mathrm{NaCl} ; 10 \mathrm{mM}$ EDTA (ethylenediaminetetraacetic acid), $100 \mathrm{mM} \mathrm{NaF}, 1 \%$ Nonidet P40 (NP40), 10\% glycerol, $2 \mathrm{mM} \mathrm{Na3VO} 4$, and $1 \mathrm{mM}$ Pefablock SC, $50 \mu \mathrm{g} / \mathrm{mL}$ aprotinin, $50 \mu \mathrm{g} / \mathrm{mL}$ leupeptin, $50 \mu \mathrm{g} / \mathrm{mL}$ bacitracin, and $50 \mu \mathrm{g} / \mathrm{mL}$ iodoacetamide as protease inhibitors. Immunoprecipitations with anti-HA antibody and protein G-sepharose beads (Sigma, Zwijndrecht, The Netherlands) and subsequent Western blotting were performed as described previously. ${ }^{7}$ Antibodies used were mouse anti-HA (Santa Cruz Biotechnology, Santa Cruz, CA), rabbit anti-SHP-2 (Santa Cruz Biotechnology), mouse anti-green fluorescent protein (Roche, Almere, The Netherlands), and rabbit anti-STAT5B (Santa Cruz Biotechnology)

\section{Results}

\section{Reduced G-CSF-induced SOCS3 expression in gcsfr-d715 mice}

SOCS proteins are under the transcriptional control of STATs. ${ }^{41,43,44,78}$ Because G-CSF-R-d715 shows altered activation of STAT3 and STAT5 compared with WT G-CSF-R, ${ }^{27,31}$ we first investigated expression of SOCS1 and 3 in WT versus gcsfr-d715 mice under steady-state conditions and after daily treatment of the animals with G-CSF for 4 days. As shown in Figure 2A, steadystate levels of SOCS1 in gcsfr-d715 mice and their wild-type littermates were similar. Also after G-CSF treatment, SOCS1 transcript levels in wild-type and gcsfr-d715 mice had not changed dramatically. A slight (2.5-fold) increase in SOCS1 mRNA levels in gcsfr-d715 mice compared with wild-type animals was noted (Figure 2A). In contrast, SOCS3 expression was strongly (greater than 12-fold) induced by G-CSF in WT mice, while expression levels in gcsfr- $d 715$ mice reached only about $30 \%$ of these levels both after G-CSF stimulation and in steady state (Figure 2B). In addition, we isolated bone marrow cells from untreated WT and d715 animals and stimulated them with G-CSF in vitro. As shown in Figure 2C, stimulation with G-CSF gives a strong induction of SOCS3 mRNA. Again, SOCS3 transcript levels are reduced in G-CSF-R-d715 cells upon stimulation with G-CSF. This demonstrates that the reduced up-regulation of SOCS3 mRNA also occurs outside the bone marrow compartment and is due to altered signaling in the G-CSF-R-d715 cells. These results establish that SOCS3 is the principal SOCS protein induced by G-CSF and that C-terminal truncation of the G-CSF-R results in a significantly reduced ability of the receptor to induce SOCS3. 

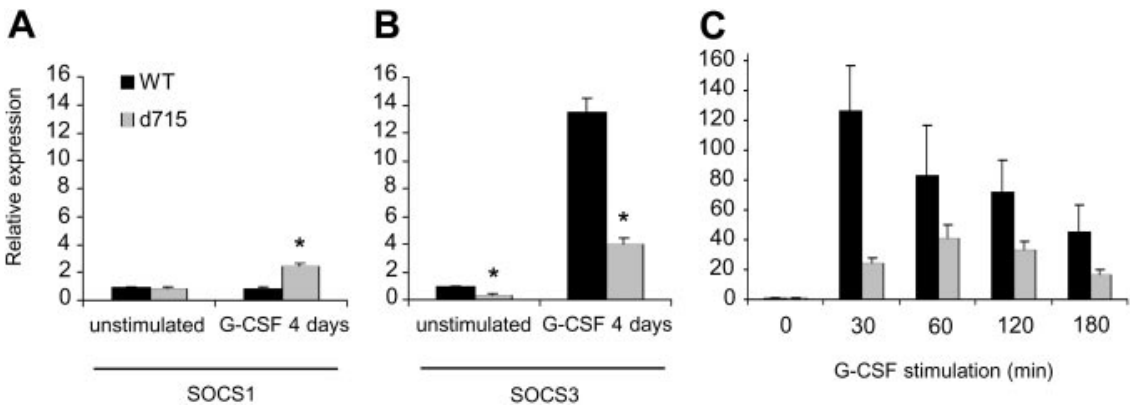

Figure 2. Reduced SOCS3 but not SOCS1 expression in gcsfr-d715 mice. WT mice and gcsfr-d715 littermates were injected with vehicle or G-CSF for 4 consecutive days. (A-B) RNA was isolated, and SOCS1 (A) and SOCS3 (B) transcript levels were measured by quantitative RT-PCR. Expression of ribonuclease inhibitor was used for normalization of the data. SOCS levels were expressed relative to untreated WT mice. Data shown are mean + standard error of the mean (SEM) of 3 experiments. ${ }^{*}$ Difference between WT and G-CSF-R-d715 is significant; $P<.05$ by Student $t$ test. (C) SOCS3 expression in bone marrow cells deprived of growth factor for 4 hours and then stimulated with G-CSF in vitro for the indicated times. SOCS3 levels were expressed relative to growth factor-deprived cells. Data shown are mean + SEM of 4 experiments; differences between WT and G-CSF-R-d715 are significant (Student $t$ test, $P<.05$ ).

\section{G-CSF-R truncation relieves the suppressive effects of SOCS3 on G-CSF-induced STAT5 but not STAT3 activation}

We next studied the consequences of SOCS3 expression on G-CSF-induced activation of STAT5 and STAT3 luciferase reporter constructs in HEK293 cell transfectants expressing either WT G-CSF-R or G-CSF-R-d715. G-CSF-induced STAT5 activity by WT G-CSF-R was dose dependently inhibited by SOCS3 (Figure 3A). In contrast, STAT5 activity induced by G-CSF-Rd715 was not affected, even at the highest SOCS3 expression levels. This finding can be reconciled with recent reports showing that Tyr729 in G-CSF-R is the major docking site for SOCS3, a residue that is lacking in G-CSF-R-d715. ${ }^{35,36}$ Surprisingly, STAT3 activation by G-CSF-R-d715 remained highly sensitive to inhibition by SOCS3 (Figure 3B). These data establish that Tyr729, while essential for down-regulation of STAT5, is dispensable for the inhibitory effects of SOCS3 on G-CSF-induced STAT3 activation.

\section{Differential effects of SOCS3 on G-CSF-R-d715-mediated STAT3 and STAT5 activation are not due to distinct involvement of upstream tyrosine kinases}

G-CSF-R activates the Janus kinases Jak1, Jak2, and Tyk2, but also the Src kinases Lyn and Hck. ${ }^{6-8,10,11}$ Both the Jak and Src kinases can phosphorylate STAT proteins in hematopoietic cells. ${ }^{81,82}$ Importantly, SOCS proteins differentially affect these kinases: in contrast to Jak kinases, the Src kinase Lyn is insensitive to SOCS-mediated inhibition. ${ }^{83}$ We considered a possible scenario in which Jak and Src kinases are differentially involved in the activation of STAT3
A

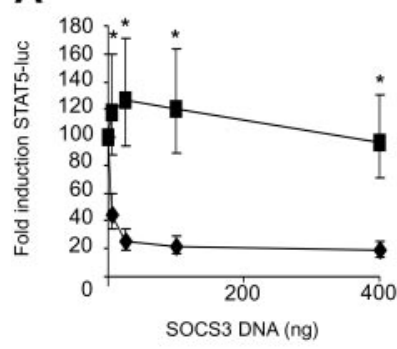

B

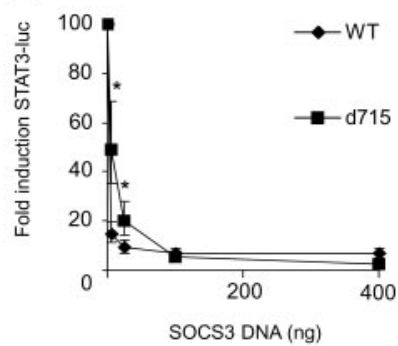

Figure 3. STAT5 activation by G-CSF-R-d715 is insensitive to inhibition by SOCS3, whereas STAT3 activation is not. HEK293 cells transfected with STAT5 or STAT3 luciferase reporter constructs were stimulated with G-CSF for 6 hours and assayed for luciferase activity. G-CSF-induced STAT5 (A) or STAT3 (B) luciferase reporter activity in the absence of SOCS3 was set at $100 \%$. Data are expressed as mean $+95 \%$ confidence interval of 4 independent experiments. *Differences between WT and G-CSF-R-d715 are significant; $P<.01$. and STAT5. In that hypothetical context, G-CSF-induced STAT5 activation by G-CSF-R-d715 would become insensitive to SOCS because, as a result of the truncation of the receptor C-terminus, involvement of Src activity in the activation of STAT5 might become prevalent. To investigate this possibility, we performed the STAT reporter experiments in the presence of the Jak inhibitor WHI-P154 or the Src inhibitor PP-2. ${ }^{84,85}$ Both STAT3- and STAT5-induced luciferase activity was inhibited by WHI-P154, but not by PP-2, indicating that Jak, but not Src-kinase activity is essential for G-CSF-induced activation of both STAT3 and STAT5 by WT G-CSF-R (Figure 4). Notably, this remained essentially unchanged when activation was induced via G-CSF-R-d715. Although PP-2 slightly reduced STAT5 activation, G-CSF-R-d715 signaling was still completely dependent on Jak activity. These results thus exclude the possibility that, in the case of G-CSF-Rd715, the loss of SOCS-mediated inhibition of STAT5 signaling is caused by altered involvement of tyrosine kinases (eg, Lyn instead of Jak) as a consequence of the receptor truncation. We have previously published data from electrophoretic mobility shift assays (EMSAs) showing that activation of STAT3 by G-CSF-Rd715 is decreased compared with WT G-CSF-R when measured between 0 and 60 minutes after stimulation. This is due to the partial lack of STAT3 recruitment mechanisms. ${ }^{31}$ We observed that activation of STAT3 by G-CSF-R-d715 in the luciferase reporter assay was increased relative to WT G-CSF-R (Figure 4B). This could be directly linked to the defective receptor internalization of G-CSF-R-d715 (data not shown). Apparently, the internalization defect of G-CSF-R-d715 causes reduced off-switch of signaling, which results in increased accumulation of luciferase activity during the 6-hour time period of the experiment. However, despite
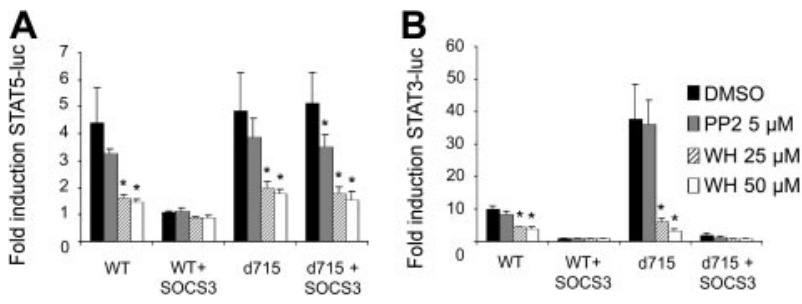

Figure 4. Effects of Jak and Src inhibitors on STAT5 and STAT3 activation by WT G-CSF-R and G-CSF-R-d715. Luciferase assays were performed as in Figure 3 . One hour before initiation of STAT5 (A) and STAT3 (B) luciferase reporter assays, the Src inhibitor PP-2 or the Jak inhibitor WHI-P154, dissolved in DMSO, was added to the cells at the concentrations indicated. Solvent control cells were treated with DMSO only. Data are expressed as mean + SEM of 3 independent experiments. ${ }^{*}$ Difference with DMSO-treated control of same group is significant; $P<.05$. 


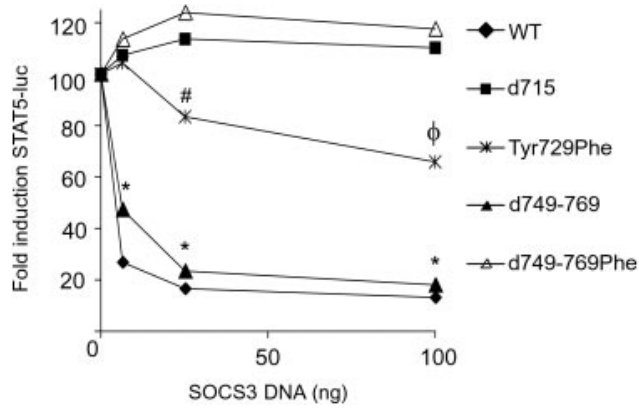

Figure 5. Reduced internalization of truncated G-CSF-R mutants alleviates inhibition by SOCS3 only in the absence of Tyr729. Comparison of G-CSF-R mutants with normal (WT and Tyr729Phe) and defective (d715, d749-769, and d749-769Phe) internalization kinetics for sensitivity to SOCS3-mediated inhibition of STAT5 luciferase reporter activity. STAT5 luciferase reporter assay was performed as described in Figure 3. Data are expressed as mean of at least 2 independent experiments with triplicate measurements. *Difference between WT and d749-769 versus all other G-CSF-R mutants is significant, $P<.01$. \#Difference between Tyr729Phe and d749-769, $P=0.07$. $\phi$ Difference between TyrY729Phe versus $\mathrm{d} 715$ and d749-769Phe is significant; $P<.02$.

this increased STAT3 activation, this signal remains fully sensitive for inhibition by SOCS3 (Figure 3B,4B).

\section{Defective G-CSF-R internalization reduces sensitivity to SOCS3 only when combined with the loss of Tyr729}

G-CSF-R-d715-induced STAT5 activation was considerably more resistant to the suppressive effects of SOCS3 than was a full-length G-CSF-R mutant lacking the SOCS3 docking site Tyr729 (eg, G-CSF-R-Tyr729Phe) (Figure 5). This could be suggestive of a second, tyrosine-independent mechanism of SOCS3 recruitment via the G-CSF-R C-terminus or relate to defective ligand-induced internalization of G-CSF-R-d715.27,31,86 To directly address this issue, we compared the SOCS3 sensitivity of WT G-CSF-R and G-CSF-R-d715 with that of G-CSF-R-d749-769 (Figure 1A). This mutant is as defective in internalization as G-CSF-R-d715, owing to the lack of 2 internalization domains. ${ }^{32}$ As shown in Figure 5, SOCS3 inhibits G-CSF-R-d749-769 as effectively as WT GCSF-R, indicating that the loss of receptor internalization per se does not alleviate STAT5 inhibition by SOCS3. However, mutation of SOCS3 recruitment site Tyr729 in this internalization-defective mutant (G-CSF-R-d749-769Phe) resulted in a complete loss of SOCS3-mediated STAT5 inhibition. These results provide evidence for 2 distinct mechanisms of SOCS3 recruitment to the G-CSF-R, one that is independent of internalization (via Tyr729) and one that requires internalization. Possibly this latter mechanism involves direct interaction of SOCS3 to Jaks. Both of these mechanisms are disrupted in G-CSF-R-d715 (Figure 5).

\section{Tyr729 is a combined recruitment site for SOCS3 and SHP-2}

For a number of receptors, it has been demonstrated that SOCS3 recruitment sites are also SHP-2 docking sites. ${ }^{57-60}$ By combined immunoprecipitation (IP) and Western-blotting (WB), we tested whether SHP-2 binds to Tyr729 of G-CSF-R. We performed these experiments with GFP-tagged receptor constructs and used antiGFP antibodies for immune detection. The GFP-tagged receptors were shown to behave identically to the untagged receptors with respect to proliferation, differentiation, and activation of STAT3 and STAT5. ${ }^{32}$ Stimulation with G-CSF induced coimmunoprecipitation of SHP-2 with the WT G-CSF receptor (Figure 6A, lanes 1-2). Truncation of the receptor at aa 715 resulted in a complete loss of the interaction with SHP-2 whereas truncation at aa $735 \mathrm{did}$ not affect the SHP-2-G-CSF-R interaction (Figure 6, lanes 3-4). This result strongly supports the notion that Tyr729, the only tyrosine present in this region, is a binding site for SHP-2. To determine the relative contribution of Tyr729 to SHP-2 recruitment to full-length G-CSF-R, we also tested G-CSF-R mutant d715-735 (Figure 1) in coimmunoprecipitations. Although this mutant demonstrated a significantly reduced SHP-2 binding compared with WT G-CSF-R, it clearly bound more SHP-2 than did G-CSF-R-d715 (Figure 6, lane 5). Taken together, these results support the notion that Tyr729 of G-CSF-R forms a combined SOCS3 and SHP-2 recruitment site and show that an alternative mechanism of SHP-2 binding to the C-terminal region (aa's 737-813) of G-CSF-R exists. The latter mechanism possibly involves recruitment of SHP-2 via Tyr764. ${ }^{20}$

\section{Tyr729 of the G-CSF-R is required for the formation of a SHP-2-STAT5 complex}

It was recently shown that SHP-2 can interact with STAT5, resulting in dephosphorylation and inactivation of STAT5. ${ }^{87,88}$ Given the requirement of Tyr729 for recruitment of SHP-2 to the G-CSF-R, we investigated if the formation of a SHP-2-STAT5 complex would be dependent on the presence of Tyr729 of the G-CSF-R as well. As shown in Figure 6B (lanes 1-4), stimulation of the WT and G-CSF-R-d735 indeed results in coimmunoprecipitation of STAT5 with SHP-2. However, in the absence of Tyr729, formation of this complex is disrupted (lanes 5-6), demonstrating the importance of Tyr729 of the G-CSF-R for the formation of a SHP-2-STAT5 complex.

\section{Discussion}

We investigated whether, and by which mechanism(s), altered susceptibility to the inhibitory effects of SOCS proteins contributes to the hyperproliferative signaling of G-CSF-R C-terminal truncation mutants, which are frequently found in SCN patients with
A

Figure 6. SHP-2 associates with distinct regions of G-CSF-R, and Tyr729 is required for the formation of a SHP-2-STAT5 complex. (A) HA-SHP-2 IPs of Phoenix E cells expressing GFP-tagged G-CSF-R mutants. Cells were starved overnight (-) and stimulated for 10 minutes with G-CSF. As a control, expression of GFP-G-CSF-R and SHP-2 in whole cell lysate (WCL) is shown in the lower 2 panels. (B) HA-SHP-2 IPs of Phoenix E cells expressing different G-CSF-R mutants and STAT5 were performed as described for Figure 6A. Lane 7 is an HA-IP in the absence of HA-SHP-2, demonstrating the specificity of the IP.

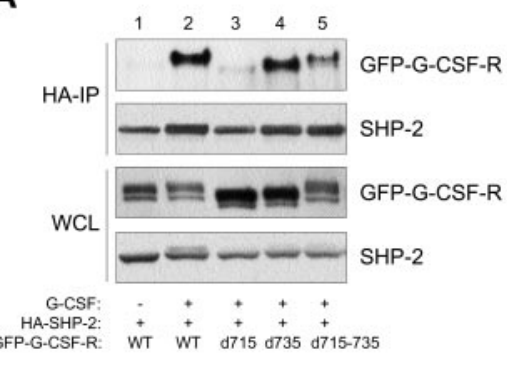

B

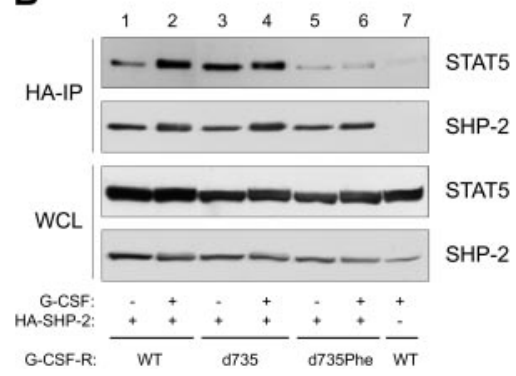


disease progression toward AML. ${ }^{24-26}$ We first concentrated on the regulation of expression of SOCS1 and SOCS3, which represent the SOCS family members with the most prominent negative effects on G-CSF signaling. ${ }^{74}$ Both SOCS1 and SOCS3 promoters contain STAT-binding sites, ${ }^{41,43,44,80}$ and dominant negative STAT3 blocks SOCS1 as well as SOCS3 mRNA expression, indicating that both SOCS genes are transcriptional targets of STAT3. ${ }^{41}$ STAT1 also binds to the SOCS3 promoter, ${ }^{44}$ but STAT3 is the major STAT protein involved in SOCS3 induction by G-CSF. This was demonstrated most clearly in conditional STAT3 knock-out mice that also lacked G-CSF-induced up-regulation of SOCS3. ${ }^{45}$ Because the loss of the G-CSF-R C-terminus affects both intensity and duration of the activation of STATs, we investigated SOCS1 and SOCS3 transcript levels in wild-type and $g c s f r$ - $d 715$ mice, both before and after 4 days of G-CSF treatment of the mice. G-CSF treatment of wild-type animals did not affect SOCS1 transcript levels. Receptor truncation resulted in a modest increase in SOCS1 expression after G-CSF stimulation, possibly owing to the increased STAT5 activation by G-CSF-R-d715. ${ }^{31}$ This is in agreement with data showing that interleukin 3 (IL-3)-induced SOCS1 expression is almost completely abrogated in the presence of dominant negative STAT5. ${ }^{89}$ SOCS3 mRNA levels, on the other hand, increased more than 12-fold in response to G-CSF. SOCS3 levels in gcsfr-d715 mice were clearly lower than in their wild-type littermates during steady state and upon stimulation with G-CSF in vivo or in vitro. These results establish that distinct regulatory mechanisms for upregulation of SOCS1 and SOCS3 expression exist in the context of the G-CSF-R. Recent studies with conditional STAT3 and SOCS3 knock-out strains demonstrated that the levels of neutrophilia in these mice in response to G-CSF treatment are remarkably similar to those observed in $g c s f r$ - $d 715$ mice..$^{28,29,45,75,76}$ This would support the hypothesis that the loss of the negative feedback loop involving STAT3-controlled induction of SOCS3 might suffice to explain the gcsfr-d715 phenotype. However, in view of previous data showing that STAT3 activation in $g c s f r$ - $d 715$ mice is only moderately and temporarily reduced, ${ }^{31}$ it is conceivable that factors other than STAT3 also contribute to G-CSF-induced SOCS3 expression.

In addition to the reduced expression of SOCS3 in the gcsfr-d715 mice, the truncated G-CSF-R lacks Tyr729, which is the major recruitment site for SOCS3. ${ }^{35,36}$ While this provided an obvious second mechanism by which the truncated G-CSF-R forms bypass the suppressive effects of SOCS3, this applied to the activation of STAT5 but not STAT3, which still remained SOCS3 sensitive (Figure 3 ). This result provides a likely explanation for the increased ratio of STAT5/STAT3 activation by G-CSF-R-d715, which has been linked to shifting the proliferation/differentiation balance toward proliferation and extended cell survival. ${ }^{31}$ Two potential explanations, differential involvement of upstream tyrosine kinases and differential sensitivity due to altered internalization kinetics, were shown, respectively, to play no or only a limited role in this study. For efficient activation of STAT3, first G-CSF-R tyrosine phosphorylation is required, which creates STAT3 recruitment sites. This indicates that activation of STAT3 is a multistep process. It is conceivable that inhibition of STAT3 is still achieved when recruitment of SOCS3 is suboptimal owing to the lack of Tyr729. In contrast, inhibition of STAT5, which is activated through direct interaction with Jak kinases,${ }^{90}$ may require optimal SOCS3 recruitment for complete inhibition. STAT5 activation by G-CSF-R mutant Tyr729Phe was inhibited to a considerable extent at the highest levels of SOCS3, which is probably mediated through low-affinity interaction of SOCS3 and Jaks.

Moreover, we think that the loss of recruitment of SHP-2 activity to Tyr729 also plays a major role in the sustained STAT5 activation by G-CSF-R-d715. It was shown for a number of cytokine receptors, for example, the leptin receptor and the shared cytokine receptor subunit gp130, that SOCS3 and SHP-2 dock to the same phosphotyrosine-based motif. ${ }^{57-60,91,92}$ Our data on the G-CSF-R (Figure 6) corroborate this and implicate Tyr729 as a common recruitment site for SOCS3 and SHP-2. Because SHP-2 was recently identified as a STAT5 phosphatase, a model can be envisaged in which impaired recruitment of SHP-2 is key to the loss of negative control of STAT5 activation. ${ }^{87,88}$ In this scenario, reduced recruitment of SHP-2 activity to the G-CSF-R would thus result in increased levels of active STAT5 complexes. Indeed, as shown in Figure 6B, G-CSF-R-Tyr729 is essential for formation of a SHP-2-STAT5 complex, which strongly argues for a scenario in which loss of Tyr729 not only affects SOCS3-mediated inhibition of STAT5 activity but also disrupts formation of a SHP-2-STAT5 complex that has been reported to contribute to STAT5 dephosphorylation.

In conclusion, our study has unveiled a new mechanism by which C-terminal truncation mutants of G-CSF-R, associated with leukemic progression of SCN, attain altered signaling abilities. While the altered signaling abilities were originally attributed mainly to defective internalization properties of G-CSF-Rd71527,31,86 the loss of negative feedback projected by SOCS3 and, possibly, SHP-2 via binding to Tyr729 of G-CSF-R have now been identified as additional signaling defects of truncated G-CSF-R. Our results fit into a model in which the combined loss of regulation by SOCS3 and SHP-2 contributes to the perturbed signaling by G-CSF-R-d715, resulting in an increased STAT5/ STAT3 activation ratio.

\section{Acknowledgments}

We thank Dr Montserrat Blazquez-Domingo for advice and help with quantitative RT-PCR, Karola van Rooyen for help with preparation of the figures, Alexandra Klomp and Dr Joanna Prasher for assistance with animal experiments, Wim van Putten for help with statistical analysis, and Dr Akihiko Yoshimura for providing SOCS3 expression plasmids.

\section{References}

1. Lieschke GJ, Grail D, Hodgson G, et al. Mice lacking granulocyte colony-stimulating factor have chronic neutropenia, granulocyte and macrophage progenitor cell deficiency, and impaired neutrophil mobilization. Blood. 1994;84:17371746

2. Liu F, Wu HY, Wesselschmidt R, Kornaga T, Link DC. Impaired production and increased apoptosis of neutrophils in granulocyte colony-stimulating factor receptor-deficient mice. Immunity. 1996;5: 491-501.
3. Zhan Y, Lieschke GJ, Grail D, Dunn AR, Cheers C. Essential roles for granulocyte-macrophage colony-stimulating factor (GM-CSF) and G-CSF in the sustained hematopoietic response of Listeria monocytogenes-infected mice. Blood. 1998; 91:863-869.

4. Bazan JF. Structural design and molecular evolution of a cytokine receptor superfamily. Proc Nat Acad Sci U S A. 1990;87:6934-6938.

5. Cosman D. The hematopoietin receptor superfamily. Cytokine. 1993;5:95-106.
6. Nicholson SE, Oates AC, Harpur AG, Ziemiecki A, Wilks AF, Layton JE. Tyrosine kinase JAK1 is associated with the granulocyte-colony-stimulating factor receptor and both become tyrosinephosphorylated after receptor activation. Proc Natl Acad Sci U S A. 1994;91:2985-2988.

7. Barge RM, de Koning JP, Pouwels K, Dong F, Lowenberg B, Touw IP. Tryptophan 650 of human granulocyte colony-stimulating factor (G-CSF) receptor, implicated in the activation of JAK2, is also required for G-CSF-mediated activation of 
signaling complexes of the p21 ras route. Blood. 1996;87:2148-2153.

8. Shimoda K, Iwasaki H, Okamura S, et al. G-CSF induces tyrosine phosphorylation of the JAK2 protein in the human myeloid G-CSF responsive and proliferative cells, but not in mature neutrophils. Biochem Biophys Res Commun. 1994;203: 922-928.

9. Shimoda K, Feng J, Murakami H, et al. Jak1 plays an essential role for receptor phosphorylation and Stat activation in response to granulocyte colonystimulating factor. Blood. 1997;90:597-604.

10. Corey SJ, Dombrosky-Ferlan PM, Zuo S, et al. Requirement of Src kinase Lyn for induction of DNA synthesis by granulocyte colony-stimulating factor. J Biol Chem. 1998;273:3230-3235.

11. Ward AC, Monkhouse JL, Csar XF, Touw IP, Bello PA. The Src-like tyrosine kinase Hck is activated by granulocyte colony-stimulating factor (G-CSF) and docks to the activated G-CSF receptor. Biochem Biophys Res Commun. 1998;251:117-123.

12. Tian SS, Lamb P, Seidel HM, Stein RB, Rosen J. Rapid activation of the STAT3 transcription factor by granulocyte colony-stimulating factor. Blood. 1994;84:1760-1764.

13. de Koning JP, Dong F, Smith L, et al. The membrane-distal cytoplasmic region of human granulocyte colony-stimulating factor receptor is required for STAT3 but not STAT1 homodimer formation. Blood. 1996;87:1335-1342.

14. Tian SS, Tapley P, Sincich C, Stein RB, Rosen J, Lamb P. Multiple signaling pathways induced by granulocyte colony-stimulating factor involving activation of JAKs, STAT5, and/or STAT3 are required for regulation of three distinct classes of immediate early genes. Blood. 1996;88:44354444.

15. Shimozaki K, Nakajima K, Hirano T, Nagata S. Involvement of STAT3 in the granulocyte colonystimulating factor-induced differentiation of myeloid cells. J Biol Chem. 1997;272:25184-25189.

16. de Koning JP, Soede-Bobok AA, Ward AC, et al. STAT3-mediated differentiation and survival and of myeloid cells in response to granulocyte colony-stimulating factor: role for the cyclin-dependent kinase inhibitor p27(Kip1). Oncogene. 2000;19:3290-3298

17. Dong F, Liu X, de Koning JP, et al. Stimulation of Stat5 by granulocyte colony-stimulating factor (G-CSF) is modulated by two distinct cytoplasmic regions of the G-CSF receptor. J Immunol. 1998; 161:6503-6509.

18. de Koning JP, Schelen AM, Dong F, et al. Specific involvement of tyrosine 764 of human granulocyte colony-stimulating factor receptor in signal transduction mediated by $\mathrm{p} 145 / \mathrm{Shc} / \mathrm{GRB} 2$ or p90/GRB2 complexes. Blood. 1996;87:132-140.

19. de Koning JP, Soede-Bobok AA, Schelen AM, et al. Proliferation signaling and activation of Shc, p21Ras, and Myc via tyrosine 764 of human granulocyte colony-stimulating factor receptor. Blood. 1998;91:1924-1933.

20. Ward AC, Smith L, de Koning JP, van Aesch Y, Touw IP. Multiple signals mediate proliferation, differentiation, and survival from the granulocyte colony-stimulating factor receptor in myeloid 32D cells. J Biol Chem. 1999;274:14956-14962.

21. Chakraborty A, Dyer KF, Cascio M, Mietzner TA, Tweardy DJ. Identification of a novel Stat3 recruitment and activation motif within the granulocyte colony-stimulating factor receptor. Blood. 1999;93:15-24

22. Ward AC, Hermans MH, Smith L, et al. Tyrosinedependent and -independent mechanisms of STAT3 activation by the human granulocyte colony-stimulating factor (G-CSF) receptor are differentially utilized depending on G-CSF concentration. Blood. 1999;93:113-124.

23. Akbarzadeh S, Ward AC, McPhee DO, Alexander WS, Lieschke GJ, Layton JE. Tyrosine residues of the granulocyte colony-stimulating factor re- ceptor transmit proliferation and differentiation signals in murine bone marrow cells. Blood. 2002; 99:879-887.

24. Dong F, Brynes RK, Tidow N, Welte K, Lowenberg B, Touw IP. Mutations in the gene for the granulocyte colony-stimulating-factor receptor in patients with acute myeloid leukemia preceded by severe congenital neutropenia [Comment appears in N Engl J Med. 1995;333:516-518]. N Engl J Med. 1995;333:487-493.

25. Dong F, Dale DC, Bonilla MA, et al. Mutations in the granulocyte colony-stimulating factor receptor gene in patients with severe congenital neutropenia. Leukemia. 1997;11:120-125.

26. Freedman $\mathrm{MH}$, Alter BP. Risk of myelodysplastic syndrome and acute myeloid leukemia in congenital neutropenias. Semin Hematol. 2002;39: 128-133.

27. Ward AC, van Aesch YM, Schelen AM, Touw IP. Defective internalization and sustained activation of truncated granulocyte colony-stimulating factor receptor found in severe congenital neutropenia/ acute myeloid leukemia. Blood. 1999;93:447-458.

28. Hermans MH, Ward AC, Antonissen C, Karis A, Lowenberg B, Touw IP. Perturbed granulopoiesis in mice with a targeted mutation in the granulocyte colony-stimulating factor receptor gene associated with severe chronic neutropenia. Blood. 1998;92:32-39.

29. McLemore ML, Poursine-Laurent J, Link DC. Increased granulocyte colony-stimulating factor responsiveness but normal resting granulopoiesis in mice carrying a targeted granulocyte colonystimulating factor receptor mutation derived from a patient with severe congenital neutropenia. J Clin Invest. 1998;102:483-492.

30. Mitsui T, Watanabe S, Taniguchi Y, et al. Impaired neutrophil maturation in truncated murine G-CSF receptor-transgenic mice. Blood. 2003;101:29902995.

31. Hermans MH, Antonissen C, Ward AC, Mayen AE, Ploemacher RE, Touw IP. Sustained receptor activation and hyperproliferation in response to granulocyte colony-stimulating factor (G-CSF) in mice with a severe congenital neutropenia/acute myeloid leukemia-derived mutation in the G-CSF receptor gene. J Exp Med. 1999;189:683-692.

32. Aarts LHJ, Roovers O, Ward AC, Touw IP. Receptor activation and 2 distinct $\mathrm{COOH}$-terminal motifs control G-CSF receptor distribution and internalization kinetics. Blood. 2004;103:571-579.

33. Endo TA, Masuhara M, Yokouchi M, et al. A new protein containing an $\mathrm{SH} 2$ domain that inhibits JAK kinases. Nature. 1997;387:921-924.

34. Cohney SJ, Sanden D, Cacalano NA, et al. SOCS-3 is tyrosine phosphorylated in response to interleukin-2 and suppresses STAT5 phosphorylation and lymphocyte proliferation. Mol Cell Biol. 1999;19:4980-4988.

35. Hortner M, Nielsch U, Mayr LM, Johnston JA, Heinrich PC, Haan S. Suppressor of cytokine signaling-3 is recruited to the activated granulocytecolony stimulating factor receptor and modulates its signal transduction. J Immunol. 2002;169: 1219-1227.

36. Hermans MHA, van de Geijn GJ, Antonissen C, et al. Signaling mechanisms coupled to tyrosines in the granulocyte colony-stimulating factor receptor orchestrate G-CSF-induced expansion of myeloid progenitor cells. Blood. 2003;101:25842590

37. Krebs DL, Hilton DJ. SOCS proteins: negative regulators of cytokine signaling. Stem Cells. 2001;19:378-387.

38. Kile BT, Alexander WS. The suppressors of cytokine signalling (SOCS). Cell Mol Life Sci. 2001; 58:1627-1635.

39. Alexander WS. Suppressors of cytokine signalling (SOCS) in the immune system. Nat Rev Immunol. 2002;2:410-416.

40. Matsumoto A, Masuhara M, Mitsui K, et al. CIS, a cytokine inducible $\mathrm{SH} 2$ protein, is a target of the JAK-STAT5 pathway and modulates STAT5 activation. Blood. 1997;89:3148-3154.

41. Naka T, Narazaki M, Hirata M, et al. Structure and function of a new STAT-induced STAT inhibitor. Nature. 1997;387:924-929.

42. Saito H, Morita Y, Fujimoto M, Narazaki M, Naka T, Kishimoto T. IFN regulatory factor-1-mediated transcriptional activation of mouse STAT-induced STAT inhibitor-1 gene promoter by IFN-gamma. J Immunol. 2000;164:5833-5843.

43. He B, You L, Uematsu K, et al. Cloning and characterization of a functional promoter of the human SOCS-3 gene. Biochem Biophys Res Commun. 2003;301:386-391.

44. Auernhammer CJ, Bousquet C, Melmed S. Autoregulation of pituitary corticotroph SOCS-3 expression: characterization of the murine SOCS-3 promoter. Proc Natl Acad Sci U S A. 1999;96: 6964-6969.

45. Lee CK, Raz R, Gimeno R, et al. STAT3 is a negative regulator of granulopoiesis but is not required for G-CSF-dependent differentiation. Immunity. 2002;17:63-72.

46. Yoshimura A, Ohkubo T, Kiguchi T, et al. A nove cytokine-inducible gene CIS encodes an $\mathrm{SH}_{2}$ containing protein that binds to tyrosine-phosphorylated interleukin 3 and erythropoietin receptors. EMBO J. 1995;14:2816-2826.

47. Ram PA, Waxman DJ. Role of the cytokine-inducible $\mathrm{SH} 2$ protein $\mathrm{CIS}$ in desensitization of STAT5b signaling by continuous growth hormone. J Biol Chem. 2000;275:39487-39496.

48. Sasaki A, Yasukawa H, Shouda T, Kitamura T, Dikic I, Yoshimura A. CIS3/SOCS-3 suppresses erythropoietin (EPO) signaling by binding the EPO receptor and JAK2. J Biol Chem. 2000;275: 29338-29347.

49. Yasukawa $\mathrm{H}$, Misawa $\mathrm{H}$, Sakamoto $\mathrm{H}$, et al. The JAK-binding protein JAB inhibits Janus tyrosine kinase activity through binding in the activation loop. EMBO J. 1999;18:1309-1320.

50. Nicholson SE, Willson TA, Farley A, et al. Mutational analyses of the SOCS proteins suggest a dual domain requirement but distinct mechanisms for inhibition of LIF and IL-6 signal transduction. EMBO J. 1999;18:375-385.

51. Narazaki M, Fujimoto M, Matsumoto $T$, et al. Three distinct domains of SSI-1/SOCS-1/JAB protein are required for its suppression of interleukin 6 signaling. Proc Natl Acad Sci U S A 1998;95:13130-13134.

52. Sasaki A, Yasukawa $H$, Suzuki $A$, et al. Cytokineinducible $\mathrm{SH} 2$ protein-3 (CIS3/SOCS3) inhibits Janus tyrosine kinase by binding through the $\mathrm{N}$ terminal kinase inhibitory region as well as $\mathrm{SH} 2$ domain. Genes Cells. 1999;4:339-351.

53. Giordanetto F, Kroemer RT. A three-dimensional model of suppressor of cytokine signalling 1 (SOCS-1). Protein Eng. 2003;16:115-124.

54. Zhang JG, Farley A, Nicholson SE, et al. The conserved SOCS box motif in suppressors of cytokine signaling binds to elongins $\mathrm{B}$ and $\mathrm{C}$ and may couple bound proteins to proteasomal degradation. Proc Natl Acad Sci U S A. 1999;96:20712076.

55. Kamura T, Sato S, Haque D, et al. The Elongin $\mathrm{BC}$ complex interacts with the conserved SOCS box motif present in members of the SOCS, ras, WD-40 repeat, and ankyrin repeat families. Genes Dev. 1998;12:3872-3881.

56. Masuhara M, Sakamoto $H$, Matsumoto $A$, et al. Cloning and characterization of novel CIS family genes. Biochem Biophys Res Commun. 1997; 239:439-446.

57. Eyckerman S, Broekaert D, Verhee A, Vandekerckhove J, Tavernier J. Identification of the Y985 and Y1077 motifs as SOCS3 recruitment sites in the murine leptin receptor. FEBS Lett. 2000;486:33-37. 
58. Nicholson SE, De Souza D, Fabri LJ, et al. Suppressor of cytokine signaling-3 preferentially binds to the SHP-2-binding site on the shared cytokine receptor subunit gp130. Proc Natl Acad Sci U S A. 2000;97:6493-6498.

59. Schmitz J, Weissenbach M, Haan S, Heinrich PC, Schaper F. SOCS3 exerts its inhibitory function on interleukin- 6 signal transduction through the SHP2 recruitment site of gp130. J Biol Chem. 2000:275:12848-12856.

60. Bjorbak C, Lavery HJ, Bates $\mathrm{SH}$, et al. SocS3 mediates feedback inhibition of the leptin receptor via Tyr985. J Biol Chem. 2000;275:40649-40657.

61. Starr R, Metcalf D, Elefanty AG, et al. Liver degeneration and lymphoid deficiencies in mice lacking suppressor of cytokine signaling-1. Proc Natl Acad Sci U S A. 1998;95:14395-14399.

62. Alexander WS, Starr R, Fenner JE, et al. SOCS1 is a critical inhibitor of interferon gamma signaling and prevents the potentially fatal neonatal actions of this cytokine. Cell. 1999;98:597-608.

63. Marine JC, Topham DJ, McKay C, et al. SOCS1 deficiency causes a lymphocyte-dependent perinatal lethality. Cell. 1999;98:609-616.

64. Naka T, Matsumoto T, Narazaki M, et al. Accelerated apoptosis of lymphocytes by augmented induction of Bax in SSI-1 (STAT-induced STAT inhibitor-1) deficient mice. Proc Natl Acad Sci U S A. 1998:95:15577-15582.

65. Metcalf D, Greenhalgh CJ, Viney E, et al. Gigantism in mice lacking suppressor of cytokine signalling-2. Nature. 2000;405:1069-1073.

66. Chong MM, Cornish AL, Darwiche R, et al. Suppressor of cytokine signaling- 1 is a critical regulator of interleukin-7-dependent CD8 + T cell differ entiation. Immunity. 2003;18:475-487.

67. Marine JC, McKay C, Wang D, et al. SOCS3 is essential in the regulation of fetal liver erythropoiesis. Cell. 1999;98:617-627.

68. Roberts AW, Robb L, Rakar S, et al. Placental defects and embryonic lethality in mice lacking suppressor of cytokine signaling 3. Proc Natl Acad Sci U S A. 2001;98:9324-9329.

69. Takahashi Y, Carpino N, Cross JC, Torres M, Parganas E, Ihle JN. SOCS3: an essential regulator of LIF receptor signaling in trophoblast giant cell differentiation. EMBO J. 2003;22:372-384.

70. Yasukawa $\mathrm{H}$, Ohishi M, Mori H, et al. IL-6 induces an anti-inflammatory response in the absence of SOCS3 in macrophages. Nat Immunol. 2003;4: 551-556.

71. Croker BA, Krebs DL, Zhang JG, et al. SOCS3 negatively regulates IL-6 signaling in vivo. Nat Immunol. 2003;4:540-545

72. Seki $Y$, Inoue $H$, Nagata $N$, et al. SOCS-3 regulates onset and maintenance of $\mathrm{T}(\mathrm{H}) 2$-mediated allergic responses. Nat Med. 2003;9:1047-1054

73. Starr R, Willson TA, Viney EM, et al. A family of cytokine-inducible inhibitors of signalling. Nature. 1997;387:917-921.

74. Van De Geijn GJM, Gits J, Touw IP. Distinct activities of suppressor of cytokine signaling (SOCS) proteins and involvement of the SOCS box in controlling G-CSF signaling. J Leukocyte Biol. In press.

75. Kimura A, Kinjyo I, Matsumura Y, et al. SOCS3 is a physiological negative regulator for granulopoiesis and granulocyte colony-stimulating factor receptor signaling. J Biol Chem. 2004;279:69056910.

76. Croker BA, Metcalf D, Robb L, et al. SOCS3 is a critical physiological negative regulator of G-CSF signaling and emergency granulopoiesis. Immunity. 2004;20:153-165.

77. Morgenstern JP, Land H. Advanced mammalian gene transfer: high titre retroviral vectors with multiple drug selection markers and a complementary helper-free packaging cell line. Nucleic Acids Res. 1990;18:3587-3596.

78. Green S, Issemann I, Sheer E. A versatile in vivo and in vitro eukaryotic expression vector for protein engineering. Nucleic Acids Res. 1988;16: 369.

79. Mui AL, Wakao H, Kinoshita T, Kitamura T, Miyajima A. Suppression of interleukin-3-induced gene expression by a C-terminal truncated Stat5: role of Stat5 in proliferation. EMBO J. 1996;15: 2425-2433.

80. Paul C, Seiliez I, Thissen JP, Le Cam A. Regulation of expression of the rat SOCS-3 gene in hepatocytes by growth hormone, interleukin- 6 and glucocorticoids mRNA analysis and promoter characterization. Eur J Biochem. 2000;267:5849 5857.

81. Klejman A, Schreiner SJ, Nieborowska-Skorska $\mathrm{M}$, et al. The Src family kinase Hck couples BCR
ABL to STAT5 activation in myeloid leukemia cells. EMBO J. 2002;21:5766-5774.

82. Okutani Y, Kitanaka A, Tanaka T, et al. Src directly tyrosine-phosphorylates STAT5 on its activation site and is involved in erythropoietin-induced sig naling pathway. Oncogene. 2001;20:6643-6650.

83. Ohya K, Kajigaya S, Yamashita Y, et al. SOCS-1/ JAB/SSI-1 can bind to and suppress Tec proteintyrosine kinase. J Biol Chem. 1997;272:2717827182.

84. Sudbeck EA, Liu X-P, Narla RK, et al. Structurebased design of specific inhibitors of Janus kinase 3 as apoptosis-inducing antileukemic agents. Clin Cancer Res. 1999;5:1569-1582.

85. Hanke JH, Gardner JP, Dow RL, et al. Discovery of a novel, potent, and Src family-selective tyrosine kinase inhibitor: study of Lck- and FynT dependent T cell activation. J Biol Chem. 1996; 271:695-701.

86. Hunter MG, Avalos BR. Deletion of a critical internalization domain in the G-CSFR in acute myelogenous leukemia preceded by severe congenital neutropenia. Blood. 1999;93:440-446.

87. Chen $Y$, Wen R, Yang S, et al. Identification of Shp-2 as a Stat5A phosphatase. J Biol Chem. 2003;278:16520-16527.

88. Yu CL, Jin YJ, Burakoff SJ. Cytosolic tyrosine dephosphorylation of STAT5. Potential role of SHP-2 in STAT5 regulation. J Biol Chem. 2000; 275:599-604.

89. Monni R, Santos SC, Mauchauffe M, et al. The TEL-Jak2 oncoprotein induces Socs1 expression and altered cytokine response in $\mathrm{Ba} / \mathrm{F} 3$ cells. Oncogene. 2001;20:849-858.

90. Fujitani $Y$, Hibi M, Fukada T, et al. An alternative pathway for STAT activation that is mediated by the direct interaction between JAK and STAT. Oncogene. 1997;14:751-761.

91. Huyer G, Li ZM, Adam M, Huckle WR, Ramachandran $\mathrm{C}$. Direct determination of the sequence recognition requirements of the $\mathrm{SH} 2$ domains of SH-PTP2. Biochemistry. 1995;34:1040 1049.

92. De Souza D, Fabri LJ, Nash A, Hilton DJ, Nicola NA, Baca M. SH2 domains from suppressor of cytokine signaling-3 and protein tyrosine phosphatase SHP-2 have similar binding specificities. Biochemistry. 2002;41:9229-9236. 